\title{
Managed Care Penetration in Outpatient Substance Abuse Treatment Units
}

\author{
Jeffrey A. Alexander \\ Christy Harris Lemak \\ University of Michigan
}

\begin{abstract}
The authors present the first nationally representative data on managed care penetration in the outpatient substance abuse treatment (OSAT) sector. Thirty-eight percent of all OSAT units were involved in some form of managed care in 1995, with 22 percent of their client base covered by managed care. There is also variation in managed care penetration and activity across different types of treatment units. Private for-profit units are involved in managed care to a greater extent than are public and private, not-for-profit organizations. Units affiliated with a hospital have greater participation and penetration than other units. Smaller OSAT facilities have a disproportionately large percentage of their client base in managed care arrangements. Finally, private managed care arrangements are more prevalent, more evenly distributed across organizational types, and represented in larger numbers than are public sources of managed care.
\end{abstract}

The magnitude and costs of substance abuse treatment are growing concerns of employers, as well as public and private insurers (Rice and Miller 1993; Regier et al. 1993). This has led to significant growth in managed care for mental health and substance abuse, with an estimated 142 million people with managed behavioral health benefits and 124 million in specialty managed behavioral health programs at the end of 1995 (Open Minds 1996; Institute of Medicine 1997).

Advocates of managed care claim that it holds the promise of curbing the sharply escalating costs of substance abuse treatment through curtailing

This research was supported by grants 5R01-DA03272 and 5R01-DA087231 from the National Institute on Drug Abuse. This article, submitted to Medical Care Research and Review on February 20, 1997, was revised and accepted for publication on August 10, 1997.

Medical Care Research and Review, Vol. 54 No. 4, (December 1997) 490-507

(C) 1997 Sage Publications, Inc. 
unnecessary utilization and ineffective treatment practices. They also suggest that managed care may expand access to substance abuse treatment services for those who need them. Critics of managed care argue that it reduces the effectiveness of care by placing constraints on treatment practices in an area that requires considerable discretion on the part of providers (Mechanic, Schlesinger, and McAlpine 1995; Boyle and Callahan 1995). Such questions are often played out at the point of service delivery-the treatment providers. As policymakers are enjoined to debate and evaluate the relative merits and disadvantages of managed behavioral care, they must have a basic and accurate understanding not only of the extent to which substance abuse treatment is covered by managed care but also how and in what form. At present, these questions have only been answered through anecdotal reports and case study research of individual substance abuse treatment providers (Morse 1995; Gelber 1996; French et al. 1995).

Understanding the role of managed care in substance abuse treatment is complex. First, most substance abuse treatment programs operate in an extremely fragmented financing environment. Treatment units face a myriad of different types of funding -including federal, state, and private sourceseach with potentially different eligibility requirements and payment mechanisms (Zarkin et al. 1995). Although Medicaid HMOs, private HMOs, and PPOs are considered the most common "types" of managed care organizations with linkages to substance abuse treatment programs, others may have a significant presence as well, including employee assistance plans, direct contracts with self-insured firms, and state substance abuse agencies.

Furthermore, the relationships between managed care organizations and treatment providers vary widely in their organizational structure and form. For many providers, managed care arrangements may range from formal, clearly articulated, negotiated contracts with HMOs to informal, ad hoc requirements made by managed indemnity plans at the time of client treatment. These managed care arrangements all include, to a varying degree, what might be considered managed care practices, including precertification, utilization review, case management, and other requirements for administration and treatment (Essock and Goldman 1995; Garnick et al. 1994).

Adding further complexity to the issue of managed care and substance abuse treatment providers is the possibility that managed care is unequally represented among different types of provider units. For example, private for-profit units may be more likely than public units to have higher levels of managed care because they are able to target clients covered by both private and public managed care plans.

In this article, we examine managed care penetration among outpatient substance abuse treatment (OSAT) units and present data to suggest the 
degree to which managed care activities vary across different types of these units, including control status (private not-for-profit, private for-profit, or public), organizational affiliation (hospital, mental health center, or freestanding), organizational size, and geographic location. Although substance abuse treatment services may be provided in a variety of settings such as inpatient units and therapeutic communities, specialized outpatient units are the predominant form of delivery, accounting for nearly 1 million clients in treatment each day (Substance Abuse and Mental Health Services Administration 1995).

\section{NEW CONTRIBUTION}

To examine managed care penetration in the outpatient substance abuse treatment sector, we use data from the only nationally representative study of outpatient substance abuse treatment organizations, the 1995 National Drug Abuse Treatment System Survey (NDATSS). This article contributes to the literature on managed behavioral care because it includes detailed information about the relationships among treatment providers and various managed care organizations, including data on the percentage of clients and revenues covered by managed care, the number of various arrangements in force, and unit involvement in public and private managed care programs. This article is the first to chronicle these specific relationships at a national level.

\section{MANAGED CARE}

Because of the great variety in the structure and form of treatment unit involvement with managed behavioral care, we define managed care broadly to include any contractual and noncontractual arrangements that impose treatment guidelines or restrictions that must be followed in order for OSAT units to receive payment for services provided. Contractual arrangements include formal, written contracts with HMOs and other managed behavioral care firms. Noncontractual arrangements include informal, ad hoc requirements made with insurance and other firms, often at the time of client treatment. While nearly all ( 90 percent) of the units in our study have at least one contractual managed care arrangement in force, we are interested in the wide range of "managed care" activities of these units, including those that extend beyond formal, contractual relationships with HMOs.

Furthermore, substance abuse treatment units may be involved in managed care arrangements with public organizations, private organizations, or some combination. In this study, public managed care refers to managed care arrangements between treatment providers and any public organization. Although the most frequently cited source of public managed care is Medi- 
caid, other arrangements are reported, including those with state substance abuse treatment agencies, courts and prisons, and other government entities. Private managed care refers to managed care arrangements with any private organization, including HMOs, PPOs, employee assistance programs (EAPs), insurance companies, self-insuring firms, and others.

\section{DATA AND METHOD}

This study uses data from a 1995 national survey of 618 outpatient substance abuse treatment units. We define an OSAT unit as a physical facility with resources dedicated primarily ( $>50$ percent) to treating individuals with substance abuse problems (including alcohol and other drugs) on a nonresidential basis. The sample was specifically designed to encompass the wide variety of organizations that comprise the nation's complex outpatient treatment system.

We contacted a nationally representative sample of 699 OSAT units and, of these, 618 participated for an 88 percent response rate. The sample was stratified by treatment modality (methadone/nonmethadone), control status (private for-profit, private non-profit, public), and organizational affiliation (hospital, mental health center, freestanding). OSAT units operated by the Veteran's Administration and by jails or prisons were excluded from the study.

The sampling frame for this study consists of the most complete listing available of the nation's OSAT units, a total of 32,927 treatment units (Heeringa 1996). This list, compiled by the Institute for Social Research, is a composite developed from five separate lists. These include the 1992 National Facilities Register (NFR) database from the Substance Abuse and Mental Health Services Administration, the 1992 National Drug and Alcoholism Treatment Unit Survey, the 1994 American Hospital Association Survey, the 1994 U.S. Food and Drug Administration list of licensed methadone providers, and a complete national database of businesses with a Standard Industrial Classification Code for Drug and Alcohol Treatment Services. These five databases were merged and unduplicated.

The director and clinical supervisor of each participating OSAT unit were asked to complete phone surveys. Directors provided information concerning the unit's control status, environment, finances, parent units, and managed care arrangements. Clinical supervisors provided information about staff, clients, and services provided. We used telephone survey procedures that extensive research indicates produce highly reliable and valid data (Groves et al. 1988). Details about the sample and survey methodology of this study have been published elsewhere (D'Aunno and Vaughn 1992, 1995; Wheeler, Fadel, and D'Aunno 1992; Alexander and Lemak forthcoming). 
Of the 618 interviewed units, 593 completed all sections of the survey. Of these, 236 units reported that more than 10 of their OSAT clients were covered by managed care arrangements during the most recent fiscal year. Directors of these units completed the managed care section of the survey, which included questions about different types of public and private managed care arrangements, the nature of the arrangements, the extent to which the unit participated in managed care, requirements of managed care organizations, and the perceived effects of managed care on outpatient substance abuse treatment (sample survey questions on managed care are contained in the appendix).

\section{RESULTS}

\section{LEVEL OF MANAGED CARE PENETRATION}

Table 1 describes the level of managed care penetration in our nationally representative sample of OSAT units. Thirty-eight percent of OSAT units in the sample exceeded threshold involvement in managed care (greater than 10 clients covered under managed care arrangements). For all OSAT units in the sample, these arrangements covered an average of 77 clients, representing 16 percent of their client base. It is notable that OSAT units participated in an average of 3.7 separate managed care arrangements. These arrangements can potentially include the same type of arrangement with the same type of managed care organization (e.g., three contracts, each with an HMO) or any combination of arrangements and managed care organizations (e.g., one contract with an HMO, one contract with Medicaid, and one informal arrangement with a managed indemnity plan).

\section{MANAGED CARE AND PROVIDER TYPE}

For purposes of description, we classified OSAT units by control status, affiliation with other organizations, size, and geographic location. Several striking differences are noted when examining managed care penetration across types of OSAT units. Units under for-profit control status are, by every measure, involved in managed care to a greater degree than their counterparts in the other two control status categories. Fifty-two percent of all for-profit OSAT units in our sample were operating with managed care arrangements that covered greater than 10 clients. These arrangements represent 26 percent of all OSAT clients in for-profit units. By contrast, public OSAT programs are the least involved in managed care. Only 25 percent of public units and 8 percent of their clients are involved in managed care arrangements. Private 


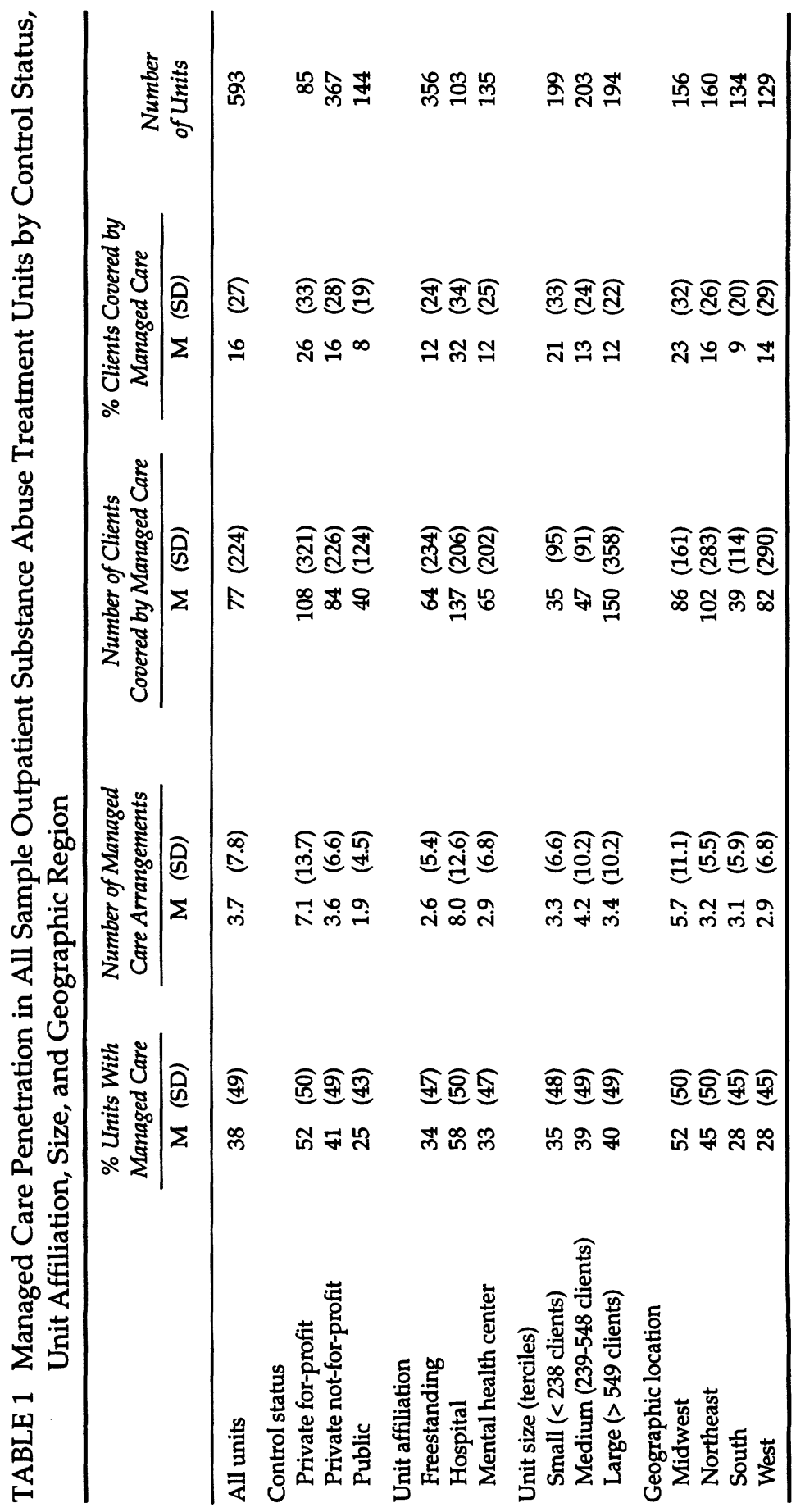


not-for-profit OSAT units occupy the middle ground between these two extremes.

Differences in managed care are also evident when comparing OSAT units across categories of organizational affiliation (defined as ownership or management by another entity). Our findings suggest that OSAT units affiliated with hospitals are more active in managed care than either OSAT units with connections to mental health centers or freestanding (unaffiliated) OSAT units. Across all four indicators of managed care penetration (percentage of units with managed care, number of managed care arrangements, number of clients, and percentage of revenues covered by managed care), hospital-affiliated units exceed the other two categories by a wide margin. OSAT units affiliated with mental health centers and freestanding OSAT units display comparable levels of managed care penetration.

Differences across OSAT size categories are concentrated primarily in the area of number and percentage of clients covered by managed care. As expected, larger OSAT units have, on average, more managed care clients compared to small and medium-sized units. However, small units have a disproportionately large percentage of their client base represented by managed care clients. On average, 21 percent of the clients of small OSAT units are covered by managed care, compared with only 13 percent and 12 percent for medium and large units, respectively.

Finally, regional comparisons indicate that the Midwest and Northeast have the largest percentages of OSAT units involved in managed care. As a percentage of clients covered by managed care, the Midwest exceeds the other three regions by a large margin. In general, the South displays the lowest level of managed care penetration in the nation.

\section{OSAT UNITS WITH MANAGED CARE}

Table 2 provides information on managed care penetration for those OSAT units that have more than 10 clients covered by managed care. Almost half of the clients (49.1 percent) and slightly less than half of the organizational revenue (45.6 percent) are covered by managed care in these units. On average, OSAT units that engage in managed care are involved with eight separate managed care arrangements. These arrangements cover an average of 191 clients.

Proportionately, slightly more clients are covered by private managed care arrangements ( 27 percent) than public managed care arrangements (22 percent). However, clients covered by private managed care are distributed among a much larger number of arrangements, on average, than clients covered under public arrangements ( 8.5 versus 1.6 , respectively). 


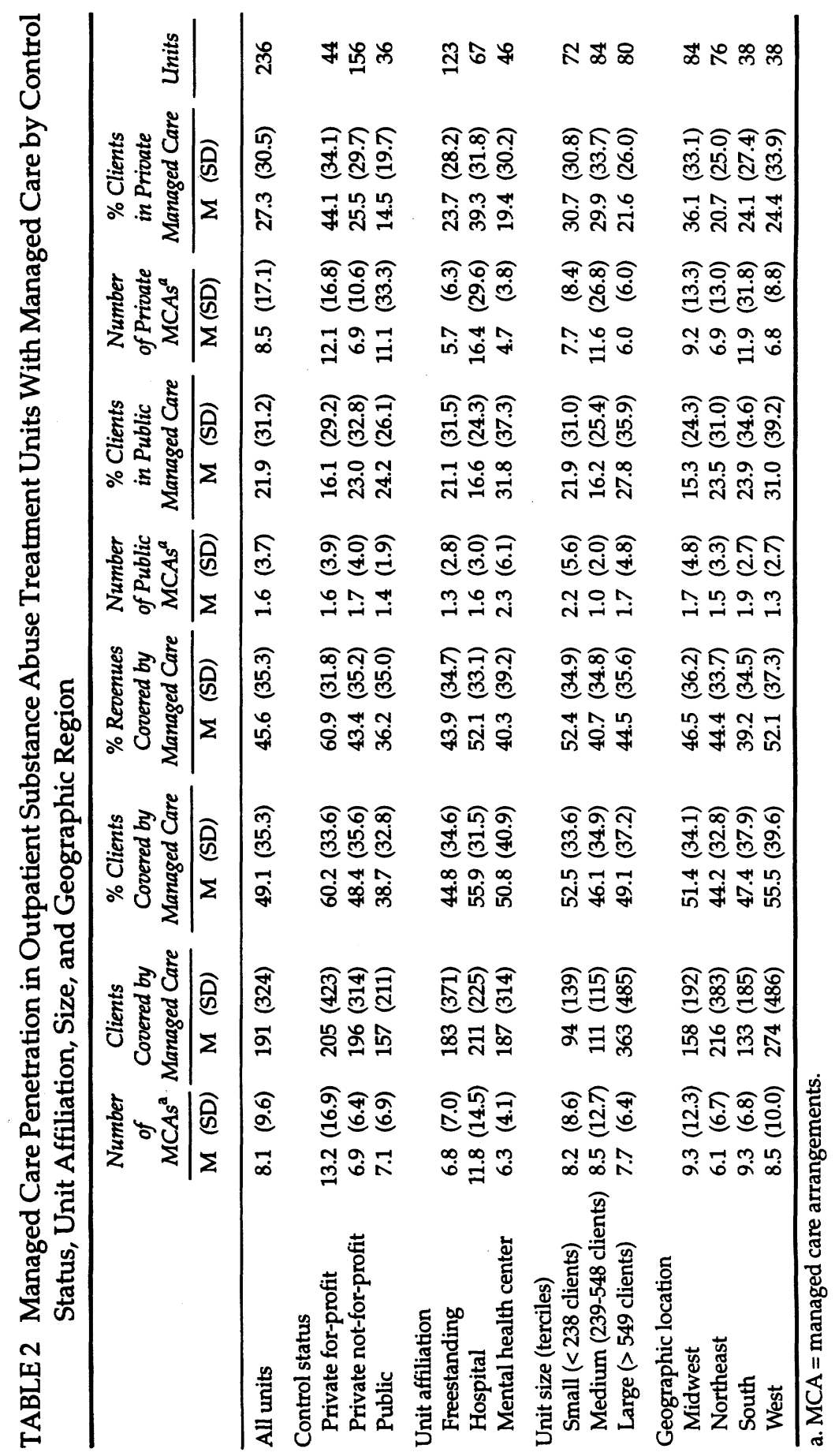


When managed care penetration for this subset of OSAT units is compared across categories of control status, affiliation, size, and geographic region, the pattern of differences is similar to those noted in Table 1. The magnitude of these differences, however, is lower across categories, suggesting that although rates of involvement in managed care may differ across types of OSAT units, those with managed care arrangements are participating at roughly similar levels. Major differences that do obtain across OSAT types are concentrated in the relative distribution of public and private managed care arrangements.

Private for-profit OSAT units, for example, are much more likely to rely on a private managed care client base than private not-for-profit or public OSAT units ( 44 percent of clients versus 25 percent and 15 percent, respectively). This disparity is less extreme across control status categories for public managed care penetration. Public and private not-for-profit OSAT units average 24 percent and 23 percent of clients covered under public managed care arrangements, while for-profit OSAT programs have 16 percent of their client base under such arrangements.

OSAT units that are affiliated with a hospital carry nearly twice the private managed care caseload as mental health center-affiliated or freestanding units and are involved with about three times the number of private managed care arrangements. Programs affiliated with mental health centers have the highest proportional public managed care caseloads ( 32 percent), followed by freestanding units (21 percent) and for-profit units (17 percent).

Variation by census region suggests that OSAT units in the Midwest are most involved with private managed care arrangements (36 percent of clients), whereas units in the West have the highest percentage of clients in public managed care ( 31 percent).

\section{PUBLIC AND PRIVATE MANAGED CARE ORGANIZATIONS}

Tables 3 and 4 present data on the frequency of types and level of activity of public and private managed care arrangements found in OSAT units. By far, the most common type of public managed care arrangement was Medicaid. Twenty percent of all sample OSAT units and 43 percent of units with managed care were involved in Medicaid managed care. All other public managed care arrangements combined were represented in 14 percent of all OSAT units and 31 percent of OSAT units with managed care. In general, OSAT units participated in relatively few public managed care arrangements-an average of 0.7 arrangements for all units and 1.6 arrangements for OSAT units meeting the managed care threshold.

By contrast, private managed care arrangements (Table 4) are much more prevalent, more evenly distributed across types, and represented in larger 
TABLE 3 Public Managed Care Arrangements of Outpatient Substance Abuse Treatment (OSAT) Organizations

$\frac{\text { All Units }}{\mathrm{M} \text { (SD) }} \frac{\begin{array}{c}\text { Units With } \\ \text { Managed Care }\end{array}}{\mathrm{M} \text { (SD) }}$

\begin{tabular}{|c|c|c|c|c|}
\hline \multicolumn{5}{|l|}{$\begin{array}{l}\text { Percentage of OSAT units with various types of public } \\
\text { managed care arrangements (MCAs) }\end{array}$} \\
\hline Medicaid & 20 & $(40)$ & 43 & $(50)$ \\
\hline Other government organization ${ }^{a}$ & 14 & (34) & 31 & $(46)$ \\
\hline State substance abuse agency & 6 & (23) & 12 & (32) \\
\hline Other state agency & 3 & (16) & 6 & $(24)$ \\
\hline Courts, prisons & 5 & $(21)$ & 10 & $(30)$ \\
\hline Other local government & 5 & $(21)$ & 10 & (30) \\
\hline Medicare & 5 & $(22)$ & 11 & $(32)$ \\
\hline Other federal programs & 3 & (16) & 6 & (23) \\
\hline \multicolumn{5}{|l|}{ Public managed care activity } \\
\hline Number of public MCAs (total) & \multicolumn{2}{|c|}{$0.7 \quad(2.6)$} & \multicolumn{2}{|c|}{$1.6(3.7)$} \\
\hline Number of public MCAs that are contractual & \multicolumn{2}{|c|}{$0.4 \quad(2.3)$} & \multicolumn{2}{|c|}{$1.2(3.4)$} \\
\hline Number of different types of public MCAs & \multicolumn{2}{|c|}{$0.5(1.7)$} & \multicolumn{2}{|c|}{$1.0(3.1)$} \\
\hline Percentage of clients covered by public MCAs & \multicolumn{2}{|c|}{$9.8(23.4)$} & \multicolumn{2}{|c|}{$21.9(31.2)$} \\
\hline Percentage of revenue covered by public MCAs & \multicolumn{2}{|c|}{$8.9(22.0)$} & \multicolumn{2}{|c|}{$19.9(29.4)$} \\
\hline Number of organizations & \multicolumn{2}{|r|}{593} & \multicolumn{2}{|c|}{236} \\
\hline
\end{tabular}

a. Because units may have managed care arrangements with more than one of the subcategories, numbers do not sum to 100 percent.

numbers in OSAT units. Although arrangements with HMOs are most frequently reported by OSAT units (68 percent of OSAT units with managed care), PPOs or exclusive provider organizations (61 percent), private insurers (67 percent), and employee assistance programs (55 percent) are also well represented among OSAT programs involved in managed care. Indeed, of all the specific types of private managed care arrangements listed in the survey, only direct contracts with self-insuring firms are represented in less than half of the OSAT units meeting the managed care threshold. ${ }^{1}$

\section{DISCUSSION}

Previous research and policy debate about managed care in the substance abuse treatment sector has focused on state financing programs; the growing number of enrollees; and the activity of large, national vendors of managed 
TABLE 4 Private Managed Care Arrangements of Outpatient Substance Abuse Treatment (OSAT) Organizations

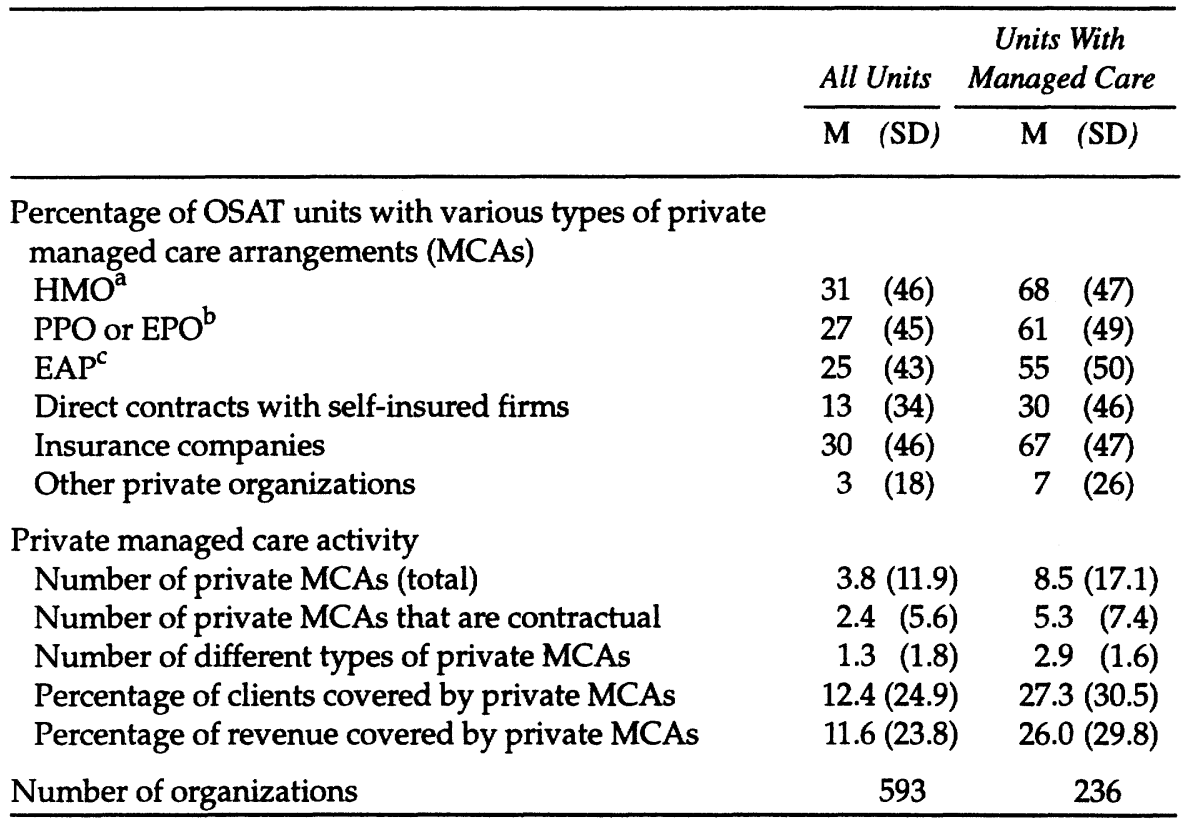

a. $\mathrm{HMO}=$ health maintenance organization.

b. $\mathrm{PPO}$ or $\mathrm{EPO}=$ preferred provider organization or exclusive provider organization.

c. $\mathrm{EAP}=$ employee assistance program.

behavioral health care. This study provides the first national data on the impact of managed care at the provider level.

Several findings from our analysis of managed care penetration in substance abuse treatment units warrant further discussion. First, we found that the majority of OSAT units are not affected by managed care, even broadly defined. Despite the rapid growth of managed behavioral care in the United States, only slightly more than one third of outpatient provider units participate in some form of managed care. There are many potential explanations for this finding. The current study took place during 1995, a time in which managed behavioral care was a relatively new concept in many markets. Alternatively, the low rate of managed care participation may suggest that either managed care organizations are restricting the delivery units from which plan members receive care or that care is provided by organizations that do not specialize in substance abuse treatment (e.g., mental health facilities, hospitals, general medical clinics). In either case, the likely consequence 
is that many OSAT units will experience severe financial difficulties if they fall outside the designated provider group of managed care organizations. As substance abuse treatment dollars continue to gravitate toward managed care, such consequences will be felt even more sharply. Furthermore, a decrease in the number of provider organizations available to those who need treatment may compound the access issues currently at the center of national policy debates (Institute of Medicine 1990, 1997).

Our findings also point to the complexity with which managed care arrangements are expressed in OSAT units. Survey results indicate that the majority of OSAT units that participate in managed care are involved with multiple arrangements and arrangement types (particularly private OSAT units). Rather than dealing with a relatively simple, single source contract for managed care, many OSAT units are faced with the administrative challenge of conforming to a series of different and even competing requirements. This may produce serious administrative burden on OSAT units-particularly small, freestanding units (Alexander and Lemak forthcoming). Recent national data suggest that the number of treatment staff in OSAT units has not increased commensurably with a greater number of clients served (D'Aunno 1996). Thus, the increased administrative burden associated with managed care must be handled by fewer staff. Faced with this zero-sum situation, many units may have to make difficult decisions to cut nontreatment services, including special programs for women and minorities and important social services for clients (Lamar and Reed 1997).

The many, often competing demands of complex managed care arrangements may have other consequences for OSAT units. For example, they may create different standards for clients covered under different plans or cause ethical dilemmas for treatment staff forced to select treatment regimens based on coverage rather than presenting conditions. A critical research question, then, is the extent to which care provided to different types of clients (e.g., managed care versus fee-for-service or managed care with stringent oversight versus managed care with little or no oversight) varies within the same treatment organization or even by the same individual provider.

Our data indicate that hospital-affiliated OSAT units are much more likely than units with other affiliations or freestanding units to have one or more managed care arrangement, and those arrangements are more likely to be with private managed care organizations. This suggests that private managed care organizations prefer to contract with providers that bring greater administrative support and experience to substance abuse treatment or that they prefer a comprehensive set of services obtainable through single-point contracts with a hospital or hospital system. Freestanding OSAT units or units affiliated with specialized units such as mental health centers cannot offer such advantages. 
These units may experience financial problems in the future, unless they can align themselves with other types of providers. Further research is needed to identify the niche these units may fill within a managed care environment, as well as the potential implications of high rates of closure for this type of OSAT facility. For example, previous research suggests that freestanding OSAT units are more likely than are other units to follow an abstinence model for treatment and are less likely to be staffed by professionals (D'Aunno, Sutton, and Price 1991). Research is needed to determine the extent to which treatment quality may be affected by increased closures among these types of units.

A consistent finding from our analysis is that private OSAT units are more "invested" in managed care than public OSAT units. Whereas this disparity can be accounted for by the relatively more rapid development of private (versus public) managed behavioral care, it is also notable that private OSAT units also treat public managed care clients. Such is not the case with public OSAT units, which tend to treat public managed care clients predominately. This may suggest that public providers of substance abuse treatment may not be as attractive to managed care organizations, even public ones such as Medicaid. As cost containment pressures grow, states may increasingly turn to the private sector for such services. Previous studies have suggested that private for-profit OSAT units operate more efficiently than other units (Wheeler, Fadel, and D'Aunno 1992). Unfortunately, it is impossible to tell from our data whether the competitive advantage of private delivery units under managed care is due to greater experience with assuming risk or to organizational cultures that value efficiency. Although risk assumption (full or partial) is not uncommon between payers and managed care organizations, it is far less common between managed care organizations and providers, where fee-based arrangements still dominate. This may suggest that price and efficiency advantages accrue to private OSAT units that make them more attractive to managed care firms. Future studies are needed to assess such differences and, perhaps more important, to assess how care is provided to substance abuse clients in private and public OSAT units.

Our study indicates that in general, OSAT units participated in relatively few public managed care arrangements. Although our sample includes units from all states, it was designed to be nationally representative rather than representative of individual states. Thus, we cannot test this proposition directly. It may be the case that wide variation in public managed care programs across states account for this result. That is, some states may have all publicly funded clients covered under a single managed care arrangement, whereas other states may have no managed care programs for public clients. Results may therefore reflect the "average" of these rather extreme statistics. 
Size of the OSAT unit was not an important discriminator of managed care activity among units with managed care. Except for absolute number of clients covered by managed care, small, medium, and large OSAT units were roughly comparable in terms of number of managed care arrangements, proportion of clients, and proportion of revenue represented by managed care. This may indicate that the ability of OSAT units to deliver care that conforms to the standards of managed care organizations may not be dictated by size of staff, scale economies, or capital-intensive technologies. Indeed, our data suggest that among all OSAT units, smaller units are more active in managed care. Managed care represents 21 percent of the client base of all small OSAT units, compared with 13 and 12 percent of medium and large units, respectively. This distribution may suggest a greater dependence on managed care funding by small OSAT units and the possibility that managed care may not result in the absorption of smaller substance abuse providers by larger ones, as it has in the general health care sector.

Although this article has attempted to describe managed care activity among several relevant categories of OSAT providers (e.g., public-private, hospital based), other comparisons may prove equally interesting in future research. For example, does the level and type of managed care activity differ in OSAT units that are owned by staff model HMOs compared to OSAT units that participate in a variety of managed care programs or with a combination of managed care and nonmanaged care funding? Or, does managed care penetration and activity differ for specialty treatment organizations compared to facilities that provide a wide range of services, including substance abuse treatment?

Because we lack trend data, many of the implications discussed above must be considered speculative. Nevertheless, these data provide important baseline information about managed care in the substance abuse sector that may inform the agendas for policymakers and researchers alike. 


\section{APPENDIX \\ Survey Questions About Managed Care}

All survey respondents were read the following information about managed care:

This section of the survey is about managed care. By managed care, we mean that organizations who pay your unit impose treatment guidelines or restrictions, such as preauthorization or utilization review. Your unit must follow these guidelines or restrictions in order to be paid for the services you provide.

Some managed care arrangements are contracts that your unit may have with managed care or insurance companies. For example, your unit may have a contract with an HMO, PPO, or other organization. Other managed care arrangements are not contractual but may impose the same guidelines or restrictions as contractual arrangements. For the purposes of this survey, please think of all your unit's managed care arrangements, both contractual and noncontractual.

1. In the most recent complete fiscal year, did your unit participate in any managed care arrangements?

2. How many different managed care arrangements did your unit participate in?

3. How many of your outpatient substance abuse treatment clients were covered by managed care arrangements?

4. Were any of these managed care arrangements with an organization your unit is owned by or part of?

5. Were any of these arrangements with your state's Medicaid program?

5a. If yes, was the arrangement with your state's Medicaid program contractual?

6. Were any of these managed care arrangements with some other government program or department, such as the state substance abuse agency, the courts, Medicare, or the county?

6a. If yes, how many of these arrangements did your unit participate in?

$6 \mathrm{~b}$. How many of these arrangements were contractual?

$6 \mathrm{cl}$. Were these arrangements with the state substance abuse agency?

$6 \mathrm{c} 2$. with other state agencies, except criminal justice agencies?

$6 \mathrm{c} 3$. with courts, prisons, jails, probation, or parole agencies?

$6 c 4$. with local government agencies?

6c5. with Medicare?

$6 \mathrm{c} 6$. with any other federal programs?

7. Were any of your unit's managed care arrangements with private insurance companies or other private organizations?

7a. If yes, how many such arrangements did your unit participate in?

$7 \mathrm{~b}$. How many of these arrangements were contractual?

$7 \mathrm{c} 1$. Of your unit's managed care arrangements with private organizations, including insurers, were the arrangements with HMOs?

7c2. with PPOs or exclusive provider organizations (EPOs)?

7c3. with employee assistance programs (EAPs)? 
7c4. were direct contracts with self-insuring firms?

7c5. with insurance companies?

7c6. with any other organizations (specify)?

8. What percentage of your unit's outpatient substance abuse clients were covered by

8a. managed care arrangements with an organization your unit is owned by or part of?

$8 \mathrm{~b}$. managed care arrangements with your state's Medicaid program?

8c. managed care arrangements with any government agency or program except Medicaid?

$8 \mathrm{~d}$. managed care arrangements with private insurers or other private organizations?

9. What percentage of your unit's outpatient substance abuse revenues were covered by

9a. managed care arrangements with an organization your unit is owned by or part of?

$9 \mathrm{~b}$. managed care arrangements with your state's Medicaid program?

9c. managed care arrangements with any government agency or program except Medicaid?

9d. managed care arrangements with private insurers or other private organizations?

\section{NOTE}

1. It is important to note that the aforementioned distinctions are based on descriptive comparisons of means or percentage values across categories. Because standard deviations are quite large in some instances, considerable variation in managed care activity may exist within particular categories. This may moderate differences across categories.

\section{REFERENCES}

Alexander, J. A., and C. H. Lemak. Forthcoming. The Effects of Managed Care on Administrative Burden in Outpatient Substance Abuse Treatment Facilities. Medical Care 35 (10).

Boyle, P. J., and D. Callahan. 1995. Managed Care and Mental Health: The Ethical Issues. Health Affairs 14 (3): 7-22.

D'Aunno, T. A. 1996. Treating Drug Abuse in America: Results from a Study of the Outpatient Substance Abuse Treatment System, 1988-1995. Ann Arbor: University of Michigan, Survey Research Center, Institute for Social Research.

D'Aunno, T. A., R. I. Sutton, and R. H. Price. 1991. Isomorphism and External Support in Conflicting Institutional Environments: A Study of Drug Abuse Treatment Units. Academy of Management Journal, 34 (3): 636-661. 
D'Aunno, T., and T. E. Vaughn. 1992. Variations in Methadone Treatment Practices: Results from a National Study. Journal of the American Medical Association 267 (2): 253-258.

1995. An Organizational Analysis of Service Patterns in Outpatient Drug Abuse Treatment Units. Journal of Substance Abuse 7:27-42.

Essock, S. M., and H. H. Goldman. 1995. States' Embrace of Managed Mental Health Care. Health Affairs 14 (3): 34-44.

French, M. T., L. J. Dunlap, D. N. Galinis, G. A. Zarkin, and J. V. Rachal. 1995. The Impact of Health Care Reforms and Managed Care on the Availability, Financing, and Costs of Substance Abuse Treatment. Report no. 271-912004. Research Triangle Park, NC: Research Triangle Institute.

Garnick, D. W., A. M. Hendricks, J. D. Dulski, K. E. Thorpe, and C. Horgan. 1994. Characteristics of Private-sector Managed Care for Mental Health and Substance Abuse Treatment. Hospital and Community Psychiatry 45 (12): 1201-1205.

Gelber, S. 1996. Managed Care and Substance Abuse: Partnership or Peril? Connection: A Newsletter Linking the Users and Producers of Drug Abuse Services Research. Washington, DC: Association for Health Services Research.

Groves, R. M., Biemer, P. P., Lyberg, L. E., Massey, J. T., Nicholls, W. L., and J. Waksberg. 1988. Telephone Survey Methodology. New York: Wiley.

Heeringa, S. G. 1996. Outpatient Drug Abuse Treatment Studies: Technical Documentation. Ann Arbor: University of Michigan, Institute for Social Research.

Institute of Medicine. 1990. Treating Drug Problems. U.S. Department of Health and Human Services, Committee for the Substance Abuse Coverage Study, ed. D. R. Gerstein and H. J. Harwood. Washington, DC: National Academy Press.

- 1997. Managing Managed Care: Quality Improvement in Behavioral Health. Committee on Quality Assurance and Accreditation Guidelines for Managed Behavioral Health Care, ed. M. Edmunds, R. Frank, M. Hogan, D. McCarty, R. Robinson-Beale, and C. Weisner. Washington, DC: National Academy Press.

Lamar, B. M., and B. G. Reed. 1997. Services for Women Clients in Outpatient Substance Abuse Treatment. Unpublished manuscript, University of Michigan, Ann Arbor.

Mechanic, D., M. Schlesinger, and D. D. McAlpine. 1995. Management of Mental Health and Substance Abuse Services: State of the Art and Early Results. Milbank Quarterly 73 (1): 19-55.

Morse, R. M. 1995. Provider Perspective: The Uneasy Alliance between Managed Care and Clinical Services. Front Lines: Linking Alcohol Research and Practice 1-2.

Open Minds. 1996. Managed Behavioral Care Market Share in the United States, 1996-1997. Gettysburg, PA: Open Minds.

Regier, D., W. Narrow, D. Rae, R. Manderscheid, B. Looke, and F. Goodwin. 1993. The de Facto U.S. Mental \& Addictive Prevalence Rates of Disorders and Services. Archives of General Psychiatry 50:85-94.

Rice, D. P., and L. K. Miller. 1993. The Economic Burden of Mental Disorders. Rockville, MD: Substance Abuse and Mental Health Services Administration. 
Sederer, L. I., and M. J. Bennett. 1996. Managed Mental Health Care in the United States: A Status Report. Administration and Policy in Mental Health 23 (4): 289-306.

Substance Abuse and Mental Health Services Administration. 1995. Overview of the National Drug and Alcoholism Treatment Unit Survey (NDATUS): 1992 and 1980-1992. Advance Rep. no. 9. Rockville, MD: Substance Abuse and Mental Health Services Administration.

Wheeler, J.R.C., H. Fadel, and T.A. D'Aunno. 1992. Ownership and Performance of Outpatient Substance Abuse Treatment Centers. American Journal of Public Health 82 (5): 711-718.

Zarkin, G. A., D. N. Galinis, M. T. French, D. L. Fountain, P. W. Ingram, and J. A. Guyett. 1995. Financing Strategies for Drug Abuse Treatment Programs. Journal of Substance Abuse Treatment 12 (6): 385-399. 